\title{
Phase-field Simulation of Effect of the Second-phase Particles with Different Geometric Orientations on Grain Evolution
}

\section{Liang MINª, Yan-Hui HOU ${ }^{a}$, Bo-Si LI ${ }^{\mathrm{a}}$, Jie PENG ${ }^{\mathrm{b}}$, Bao-Shu QIANª and Yang LIU $^{\mathrm{a}}$}

\author{
${ }^{a}$ The State Key Laboratory of Refractories and Metallurgy, Wuhan University of Science and Techn \\ ology, Wuhan, 430081,P.R.China \\ ${ }^{b}$ School of metallurgical and ecological engineering, University of Science and Technology Beijing, \\ Beijing, 10083,P.R.China
}

*houyanhui@wust.edu.cn

Keywords: Phase-field method, Grain evolution, The second phase particle, Multi-orientation, Zener pinning.

\begin{abstract}
Grain growth has significant effect on the material's microstructure and properties. In this thesis, the grain growth processes under the conditions of the second-phase particles (SPP) with different geometric orientations are studied by means of the phase-field method. The main results are as follows: 1. In the grain growth process, most of the spherical SPP locate at the intersection of crystal boundary, while stick SPP locate at the grain boundary with the same direction as the grain boundaries. 2. Under the condition that the SPP area fraction is constant, stick SPP in different orientations has little effect on the grain size. 3. Under the condition that the SPP area fraction and size are constants, there is no difference between stick SPP and spherical SPP in the pinning effect on grain growth when the area fraction of the SPP is small. 4. The SPP show a strong pinning effect on grain boundary and the limited grain radius can be expressed by Zener relations.
\end{abstract}

\section{Introduction}

Microstructures of materials largely determine their various properties of macroscopic. And the evolution of grains has a direct impact on the microstructure of material. Here we study the grain evolution with computer model.

Based on phase-field model proposed by Chen and Yang [1], Moelans et al. [2-3] first simulated grain growth in two-dimensional systems containing finely dispersed SPP. Suwa and Saito [4] simulated grain growth in three dimensional system containing finely dispersed SPP. Gao Yingjun [5] extended model proposed by Moelans et al. [3] to study pinning effects of the hard particles on grain growth. LongYongqiang.et al [6] studied phase field modeling for effects of spherical and discal SPP on grain growth. Luo Zhirong et al [7] researched effect of stick SPP with different orientations on grain growth simulated by phase-field method. This paper applies the order parameter phase-field model to simulate the effect of the second-phase particles of various orientations on grain evolution in single phase system.

\section{Phase-field Model and Numerical Solution}

According to the diffuse interface theory and field theory, the total free energy $\mathrm{F}$ can be described by all phase-field parameter and gradient coefficients, namely

$$
F=\int_{V}\left[f_{0}\left(\eta_{1}(r) \ldots \ldots \eta_{p}(r)\right)+\sum_{i=1}^{p} \frac{\kappa_{i}}{2}\left(\nabla \eta_{i}(r)\right)^{2}\right] d^{3} r
$$


Where $f_{0}\left(\eta_{1}(r) \ldots \ldots \eta_{p}(r)\right)$ is local free energy density function, $\sum_{i=1}^{p} \frac{k_{i}}{2}\left(\nabla \eta_{i}(r)\right)^{2}$ is energy gradient density, $k_{i}$ is energy gradient density coefficient.

So we carried out a series of complex calculations. The initial orientation field variables get value randomly in [-0.001, 0.001], representing the initial morphology. And two kinds of second-phase particles, spherical and stick are applied. The stick SPP has 4 spatial orientation angles and horizontal angle are respectively $0,45^{\circ}, 90^{\circ}, 135^{\circ}$, and they are equal in proportion. In this study the square grid point is

$100 \times 100$ g.p..p=36, spatial step $\Delta \mathrm{x}=2.0$, time step $\Delta \mathrm{t}=0.25, \alpha=\beta=\gamma=1, \kappa_{i}=2.0, L_{i}=1.0$.

\section{Results of Model}

Figure 3.1(a) was the image of single-phase grain growth. Fig. 3.1 (b) was the image of spherical SPP pinning on grains. Fig. 3.1(c) [8] showed black particles, Ti-Mg alloy, formed SPP in steel. From Fig. 3.1(a) (c), we know that the SPP simulated by phase-field mothed has the pinning effect on grain, and can inhibit grain growth effectively.

Figure 3.2 shows the grain evolution under the condition that the area fraction of stick and spherical SPP is 5\% and indicates that in the final microstructure obtained, most of the SPP is located at the grain boundaries, and grain boundaries become straight.

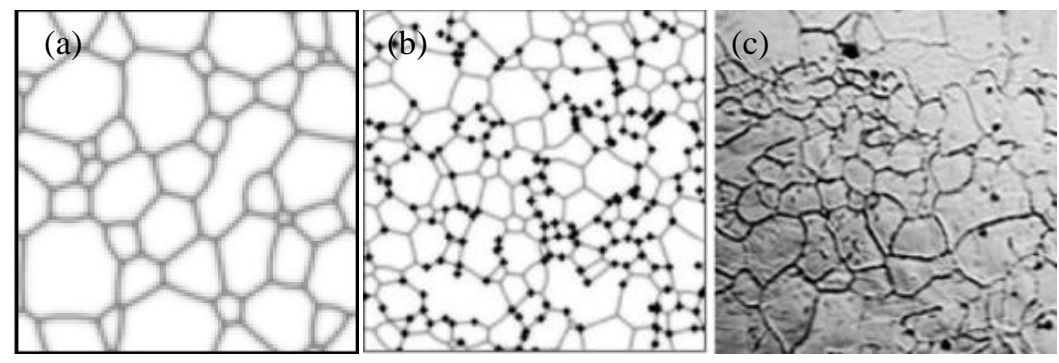

Fig. 3.1 Compares phase-field simulation results to experimental results

Figure 3.3 shows the grain microstructure containing spherical and stick SPP with different area fraction. Fig. 3.3(a) (c) can be seen, most spherical SPP are located at the trijunction, and stick SPP are mostly located in the grain boundaries and the direction are in the same with grain boundary. When spherical SPP are in trijunction, sticks SPP are in the grain boundary and their directions are same with the grain boundaries, the interface energy can be reduced the most.

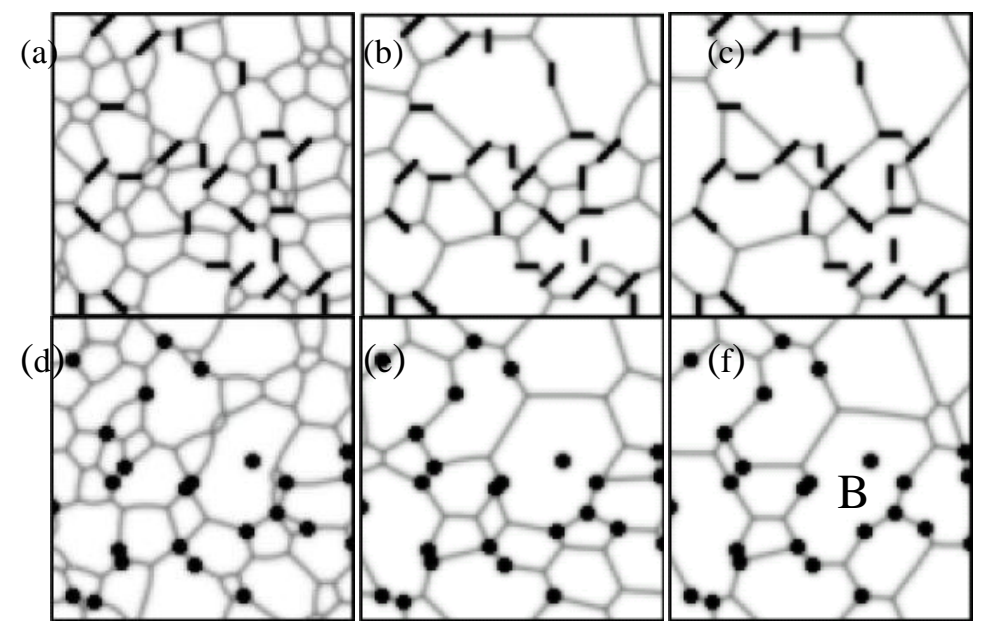

Fig. 3.2 the grain evolution under the condition that the area fraction of stick and spherical SPP is $5 \%$, where time step respectly is:

(a)1000; (b)5000; (c)20000; (d)1000; (e)5000; (f)20000 


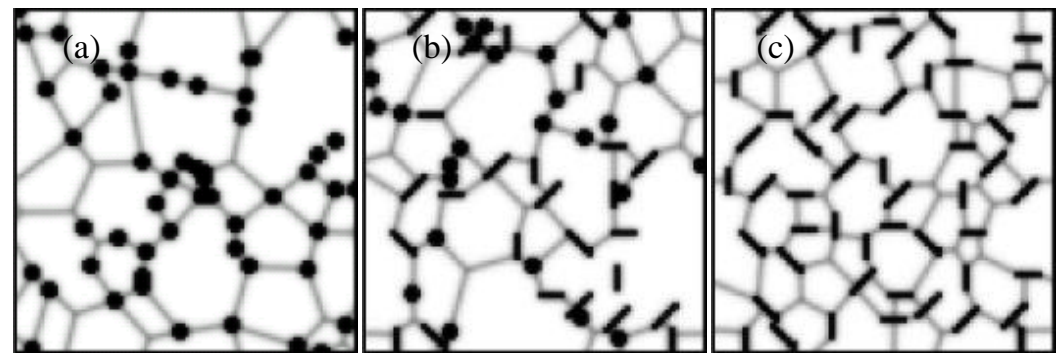

Fig. 3.3 the microstructure evolution in system containing spherical and stick SPP with different area fraction, where $\mathrm{fa}=10 \%$, time step $\mathrm{t}$ is $2.0 \times 104$

\section{Conclusions}

In this thesis, the main results are as follows:

(1)In the grain growth process, most of the spherical SPP locate at the intersection of crystal boundary, while stick SPP locate at the grain boundary with the same direction as the grain boundaries. And there appear some grains growing abnormally.

(2) Under the condition that the SPP area fraction is constant, stick SPP which was in different orientations as little effect on the grain size.

(3)Under the condition that the SPP area fraction and size are constants, there is no difference between stick SPP and spherical SPP in the pinning effect on grain growth when the area fraction of the SPP is small $(<5 \%)$.However, compared to spherical SPP, the stick SPP show more effective pinning effect when the area fraction of the SPP is large $(>5 \%)$.

(4)The SPP show a strong pinning effect on grain boundary and the limited grain radius can be expressed by Zener relations. And the simulation results are in well agreement with the MC simulation results.

\section{Acknowledgements}

This work is supported by Research Fund for the Doctoral Program of Higher Education of China (20134219120001), and Research Fund for the Returned Overseas Chinese Scholars.

\section{References}

[1] Moelans N, Blanpain B, Wollants $P$. A phase field model for the simulation of grain growth in materials containing finely dispersed incoherent second-phase particles. Acta Materialia6, 53(2005), pp.1771-1781.

[2] Moelans N, Blanpain B, Wollants P. Phase field simulations of grain growth in twodimensional systems containing finely dispersed second-phase particles. Acta Materialia4, 54(2006),pp. 1175-1184.

[3] Chen L Q, Yang W. Computer simulation of the domain dynamics of a quenched system with a large number of nonconserved order parameters: The grain-growth kinetics. Phys Rev B21, 50(1994), pp. 15752-15756.

[4] Suwa Y, Saito Y. Phase field simulation of grain growth in three dimensional system containing finely dispersed second-phase particles. Scripta Materialia4,55(2006), pp.407-410.

[5] Gao Ying-jun, Zhang Hai-lin, JIN Xing et al. Phase-field simulation of two-phasegrain growth with hard particles. Acta Metallrugica Sinica10,45(2009), pp.1190-1198.

[6] Long Yong-qiang, Liu Ping, Liu Yong, Pan Jian-sheng. Phase field modeling for effects of spherical and discal second-phase particles on grain growth. The Chinese Journal of Nonferrous Metals1,19(2009), pp.84-89. 
[7] Luo Zhi-rong, Gao Ying-jun, Qiu Hong-guang et al. Effect of stick second-phase particles with different geometric orientations on grain growth simulated by phase field method. The Chinese Journal of Nonferrous Metals12,20(2010), pp.2406-2411.

[8] Apel M, Bottger B, Rudnizki J, Schaffnit P, Steinbach I. Grain growth simulations including particle pinning using the multiphase-field concept. ISIJ international7, 49(2009), pp. 1024-1029.

[9] Song Yu. Effects of Fe-M-Ti-Mg(M=Si, Mn, Al) mult-deoxidation on the organization of steels. Master's degree thesis of Wuhan University of Science and Technology. (2011). 